\title{
Antimicrobial Properties of Various Non-Antibiotic Drugs against Microorganisms
}

Sadik Kalayci*

Department of Genetics and Bioengineering, Faculty of Engineering, Yeditepe University, Turkey

\section{Editorial}

Antibiotics and antimicrobial chemotherapeutics exist in large numbers in today's pharmaceutical market. Despite their use is becoming increasingly restricted. The reason behind such a rapid decline is largely attributed to the development of drug resistance among microorganisms. Such a phenomenon is coupled by the toxicity possessed by many antimicrobials. Multiple drug resistance among highly infective microorganisms generates a major obstacle to clinical applications in recent years. As development of a new broad range antimicrobial agent is difficult and takes several years, increasing the activity of existing antibiotics would be a future solution to this challenge. However, there is a search for newer antimicrobial agents that can overcome these drawbacks. Studies in this line have exposed the fact that several compounds, belonging to various pharmacological classes, possess moderate to powerful antibacterial activity.

There are some synthetic or natural medicinal compounds, referred as non-antibiotics, which are effective against microbial metabolism [1]. A number of non-antibiotic drugs including non-steroidal antiinflammatory drugs, calcium channel blockers and antidepressants have been reported to display biocidal or biostatic activity [2]. In addition, recent studies showed that some repellent molecules such as Picaridin and DEET, which are spread to the body of people and clothes to remove some arthropods (mosquitoes, lice, ticks, etc.), have also antimicrobial property [3].

These non-antibiotic drugs act in different manners on microbial growth. They may have direct antimicrobial activity (antimicrobial nonantibiotics), increase the efficiency of an antibiotic as given together (helper compounds), or change the pathogenicity of microorganisms or activity on the physiology such as modulating macrophage activity [4]. For example, antidepressants such as Sertraline, Paroxetine, and Fluoxetine have been shown to decrease minimum inhibition concentration (MIC) levels of several antibiotics mainly by inhibiting efflux pump activity [5-8]. Beyond acting synergistically, some psychotic drugs exhibit antimicrobial characteristics. They have been effective against gram negative and positive bacteria $[2,9,10]$, yeast [11], fungi [12], and protozoa [13].

The main limiting factor of non-antibiotic drugs to display their antimicrobial characteristics in mammalian system is that the maximum serum level remains (approx. $1 \mathrm{mg} / \mathrm{L}$ ) lower than the concentration required inhibiting microbial growth [2]. However, these levels might be sufficient to modify microbial metabolism and act synergistically with certain antibiotics $[14,15]$. For example, it has been claimed that $0.75 \mathrm{mg} / \mathrm{L}$ sertraline, lower than the tissue concentration in vivo, resulted in lack of hyphal transformation and decrease in virulence for Candida spp. [16].

Given the increasing incidence of infections and the limited efficacy of currently available antimicrobial agents, new approaches are needed. A broad spectrum of drugs produced by microorganisms might be investigated for their antimicrobial activity. On the other hand, the currently published information describes in vitro activity and in vivo efficacy in animals. There is very limited clinical information that indicates clinically relevant activity of non-antibiotics compounds in humans. In addition, there is a need to take pharmacodynamics into account in vivo. On the basis of this information, new approaches to the infection can be designed.

\section{References:}

1. Kristiansen JE (1992) The antimicrobial activity of nonantibiotics. Acta Path Microbiol Scand 100: 7-19.

2. Munoz Bellido J, Munoz Criado S, Garcia Rodriguez J (2000) Antimicrobial activity of psychotropic drugs: selective serotonin reuptake inhibitors. Int $J$ Antimicrob Agents 14: 177-180

3. Kalayci S, Demirci S, Sahin F (2014) Determination of antimicrobial properties of Picaridin and DEET against a broad range of microorganisms. World Journal of Microbiology and Biotechnology 30: 407-411.

4. Martins M, Dastidar SG, Fanning S (2008) Potential role of non-antibiotics (helper compounds) in the treatment of multidrug-resistant Gram-negative infections: mechanisms for their direct and indirect activities. Int J Antimicrob Agents 31: 198-208.

5. Amaral L, Martins A, Molnar J (2010) Phenothiazines, bacterial efflux pumps and targeting the macrophage for enhanced killing of intracellular XDRTB. In vivo 24: 409-424.

6. Bohnert JA, Szymaniak VM, Schuster S, Kern WV (2011) Efflux inhibition by selective serotonin reuptake inhibitors in Escherichia coli. J Antimicrob Chemother 66: 2057-2060.

7. Jeyaseeli L, Dasgupta A, Dastidar SG, Molnar J, Amaral L (2012) Evidence of significant synergism between antibiotics and the antipsychotic, antimicrobial drug flupenthixol. Eur J Clin Microbiol Infect Dis 31: 1243-1250.

8. Kaatz GW, Moudgal VV, Seo SM, Hansen JB, Kristiansen JE (2003) Phenylpiperidine selective serotonin reuptake inhibitors interfere with multidrug efflux pump activity in Staphylococcus aureus. Int J Antimicrob Agents 22: 254-261.

9. Jeyaseeli L, Gupta AD, Asok Kumar K, Mazumdar K, Dutta NK, et al. (2006) Antimicrobial potentiality of the thioxanthene flupenthixol through extensive in vitro and in vivo experiments. Int J Antimicrob Agents. 27: 58-62.

10. Muñioz Bellido J, Muñioz Bellido S, García Rodrfguez J (1996) In vitro activity of psychiatric drugs against Corynebacterium urealyticum (Corynebacterium group D2). J Antimicrob Chemother 37: 1005-1009.

11. Lass Flörl C, Ledochowski M, Fuchs D (2003) Interaction of sertraline with Candida species selectively attenuates fungal virulence in vitro. FEMS Immunol Med Microbiol 35: 11-15.

12. Lass Flörl C, Dierich M, Fuchs D (2001) Antifungal properties of selective serotonin reuptake inhibitors against Aspergillus species in vitro. J Antimicrob Chemother 48: 775-779.

*Corresponding author: Sadik Kalayci, Genetics and Bioengineering Department, Faculty of Engineering Yeditepe University Kayisdagi, Istanbul, Turkey, Tel: +90 (216) 578 0619; Fax: +90 (216) 578 0829; E-mail: sadik.kalayci81@gmail.com

Received July 01, 2016; Accepted July 02, 2016; Published July 05, 2016

Citation: Kalayci S (2016) Antimicrobial Properties of Various Non-Antibiotic Drugs against Microorganisms. J Bioanal Biomed 8: e142. doi:10.4172/1948593X.1000e142

Copyright: ๑ 2016 Kalayci S. This is an open-access article distributed under the terms of the Creative Commons Attribution License, which permits unrestricted use, distribution, and reproduction in any medium, provided the original author and source are credited. 
Citation: Kalayci S (2016) Antimicrobial Properties of Various Non-Antibiotic Drugs against Microorganisms. J Bioanal Biomed 8: e142. doi:10.4172/1948-593X.1000e142

13. Palit P, Ali N (2008) Oral therapy with sertraline, a selective serotonin reuptake inhibitor, shows activity against Leishmania donovani. J Antimicrob Chemother 61: $1120-1124$

14. Gunics G, Motohashi N, Amaral L, Farkas S, Molnár J (2000) Interaction between antibiotics and non-conventional antibiotics on bacteria. Int $J$ Antimicrob Agents 14: 239-242.
15. Muñoz Criado S, Muñoz Bellido J, Alonso Manzanares M, Gutiérrez Zufiaurre M, García Rodríguez J (1998) Psychotropic drugs inhibit swarming in Proteus spp. and related genera. Clin Microbiol Infect 4: 447-449.

16. Lass Flörl C, Ledochowski M, Fuchs D (2003) Interaction of sertraline with Candida species selectively attenuates fungal virulence in vitro. FEMS Immunol Med Microbiol 35: 11-15. 